\title{
First experience with robotic spleen-saving, vessel-preserving distal pancreatectomy in Singapore: a report of three consecutive cases
}

\author{
Brian KP $\underline{G o h}^{1,2}$, MMed, FRCSEd, Jen-San Wong ${ }^{1}$, MMed, FRCSEd, Chung-Yip $\underline{\text { Chan }}^{1}$, MMed, FRCSEd,

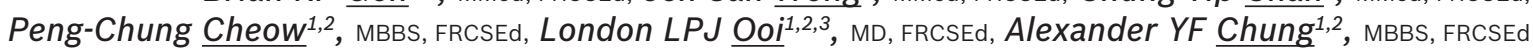

\begin{abstract}
INTRODUCTION The use of laparoscopic distal pancreatectomy (LDP) has increased worldwide due to the reported advantages associated with this minimally invasive procedure. However, widespread adoption is hindered by its technical complexity. Robotic distal pancreatectomy (RDP) was introduced to overcome this limitation, but worldwide experience with RDP is still lacking. There is presently evidence that RDP is associated with decreased conversion rate and increased splenic preservation as compared to LDP.

METHODS We conducted a prospective study on our initial experience with robotic spleen-saving, vessel-preserving distal pancreatectomy (SSVP-DP) between July 2013 and April 2014.

RESULTS Three consecutive patients underwent attempted robotic SSVP-DP. The indications were a 2.1-cm indeterminate cystic neoplasm, $4.5-\mathrm{cm}$ solid pseudopapillary neoplasm and $1.2-\mathrm{cm}$ pancreatic neuroendocrine tumour. For all three patients, the procedure was completed without conversion, and the spleen, with its main vessels, was successfully conserved. The median total operation time, blood loss and postoperative stay were 350 (range 300-540) minutes, 200 (range 50-300) $\mathrm{mL}$ and 7 (range 6-14) days, respectively. Two patients had minor Clavien-Dindo Grade I complications (one Grade A pancreatic fistula and one postoperative ileus). One patient had a Clavien-Dindo Grade IIla complication (Grade B pancreatic fistula requiring percutaneous drainage). All patients were well at the time of reporting after at least six months of follow-up.

CONCLUSION Our preliminary experience with robotic SSVP-DP confirmed the feasibility of the procedure.
\end{abstract}

Keywords: da Vinci, laparoscopic, minimally invasive, pancreatectomy, robotic

\section{INTRODUCTION}

Over the past few decades, the use of laparoscopic surgery has expanded rapidly to become the surgical approach of choice for many abdominal operations, including cholecystectomies, ${ }^{(1)}$ appendicectomies, ${ }^{(2)}$ adrenalectomies, ${ }^{(3)}$ colectomies $^{(4)}$ and gastric resections. ${ }^{(5,6)}$ However, the adoption of laparoscopic surgery in the fields of liver and pancreatic surgery has been relatively slow. ${ }^{(7,8)}$ Although the first laparoscopic liver and pancreatic resections were reported in the early 1990 s, $^{(9,10)}$ widespread adoption of such operations has been hindered by the technical complexities of these procedures, and concerns about oncological margins and risk of bleeding. ${ }^{(8)}$

Pancreatic surgery is among the most complex and challenging of all abdominal operations. ${ }^{(11)}$ Despite rapid surgical advancements, pancreatic surgery remains a challenge and is associated with a high morbidity rate worldwide, even in highly specialised and experienced tertiary centres. ${ }^{(12,13)}$ Laparoscopic pancreatic surgery was first reported by Cuschieri in 1994. ${ }^{(9)}$ Since then, a growing number of studies on laparoscopic pancreatic surgeries have been published, although it has yet to gain widespread acceptance. ${ }^{(11)}$ As distal pancreatectomy (DP) is not as technically demanding as pancreaticoduodenectomy, it is not surprising that most of the published studies are on the outcomes of laparoscopic DP (LDP). ${ }^{(14)}$ These early studies have reported the potential benefits of using the laparoscopic approach, including decreased pain and blood loss, faster recovery, and a shorter hospital stay; the laparoscopic approach is also associated with morbidity rates that are similar to those observed with the use of the open approach. ${ }^{(14,15)}$ Despite the increased adoption of LDP, there are barriers to its widespread adoption, which include the significant risk of conversion to the hand-assisted or open approach. ${ }^{(15,16)}$

Robotic surgery was introduced to overcome the shortcomings of conventional laparoscopic surgery. ${ }^{(11,15,17)}$ The robotic platform provides a three-dimensional, high-definition, magnified view of the operative field. It also provides the additional advantages of improved dexterity and precision due to increased freedom of movement and the elimination of tremors. ${ }^{(7,11)}$ The superiority of robotic surgery over conventional laparoscopy when performing complex surgical tasks has been proven in ex vivo models. ${ }^{(15)}$ However, its use in the clinical setting remains controversial, especially with regard to DP. In the first series of robotic distal pancreatectomies (RDPs), reported by Giulianotti et al in 2003, 13 patients underwent RDP. ${ }^{(18)}$ Several studies on RDP have since been reported, most of which were small case series involving less than 50 patients. ${ }^{(17)}$

${ }^{1}$ Department of Hepatopancreatobiliary and Transplantation Surgery, Singapore General Hospital, ${ }^{2}$ Duke-NUS Medical School, ${ }^{3}$ Division of Surgery, Singapore General Hospital, Singapore

Correspondence: A/Prof Brian KP Goh, Senior Consultant and Adjunct Associate Professor, Department of Hepatopancreatobiliary and Transplantation Surgery, Singapore General Hospital, 20 College Road, Academia, Singapore 169856. bsgkp@hotmail.com 
Traditionally, DP is performed together with splenectomy due to the close relationship between the pancreas, splenic artery and splenic vein. ${ }^{(19,20)}$ However, as there is increasing evidence that the spleen has an important immunological function, more surgeons are attempting to conserve the spleen when resecting left-sided, non-malignant pancreatic neoplasms. ${ }^{(19,20)}$ In general, there are two techniques for performing spleen-saving distal pancreatectomy (SS-DP). The first technique, the spleen-saving, vessel-preserving distal pancreatectomy (SSVP-DP), requires the preservation of the main splenic artery and vein. This technique is challenging due to the close relationship between the aforementioned vessels and the pancreas. Additionally, numerous small and fragile vessels enter the pancreas via these vessels. The second technique, known as Warshaw's technique, entails sacrificing the main splenic artery and vein. ${ }^{(21)}$ In this technique, segmental resection of the aforementioned vessels is performed and the spleen is left to be perfused by the short gastric and left gastroepiploic vessels. ${ }^{(19,22)}$ Although it is potentially easier to perform than SSVP-DP, Warshaw's technique is associated with increased risk of splenic infarction and left-sided portal hypertension. ${ }^{(19,22,23)}$ Hence, many authors advocate SSVP-DP as the technique of choice whenever it is technically feasible. ${ }^{(11,19,20)}$ Laparoscopic SSVP-DP is a challenging operation and inadvertent splenectomy is reported in $>50 \%$ of cases. ${ }^{(14)}$ More recently, in order to overcome the limitations of conventional laparoscopy, several authors have advocated the use of RDP to increase the rate of splenic preservation. ${ }^{(1,24)}$ However, experience with laparoscopic SSVP-DP, especially via robotic assistance, remains limited worldwide. ${ }^{(11,17)}$ We herein report our experience with RDP for robotic SSVP-DP in the first three consecutive cases that presented to us. This study aimed to determine the feasibility and safety of RDP for SSVP-DP.

\section{METHODS}

Between July 2013 and April 2014, three consecutive patients underwent attempted robotic SSVP-DP at Singapore General Hospital, Singapore. This prospective study was approved by the institution's review board. All three robotic SSVP-DPs were performed using the da Vinci® Si System (Intuitive Surgical Inc, Mountain View, CA, USA) using three robotic arms (one camera arm and two working arms). All procedures were performed by a team of surgeons comprising a da Vinci robot-accredited console surgeon and one or two bedside surgical assistants (who were also da Vinci robot-accredited or had experience in laparoscopic pancreatectomy). The surgical assistants stood between the patient's legs and/or on the right side of the patient. In all three cases, spleen preservation with conservation of the main splenic vessels was attempted. A closed suction drain was used in all three cases and the drain fluid was routinely sent for biochemical testing for amylase on postoperative Day 3. Postoperative pancreatic fistula was present when any amount of drain fluid had an amylase content that was greater than three times of the upper normal limit of serum amylase. This definition is according to the International Study Group on Pancreatic Fistula (ISGPF); grading of the pancreatic fistulas were also assigned

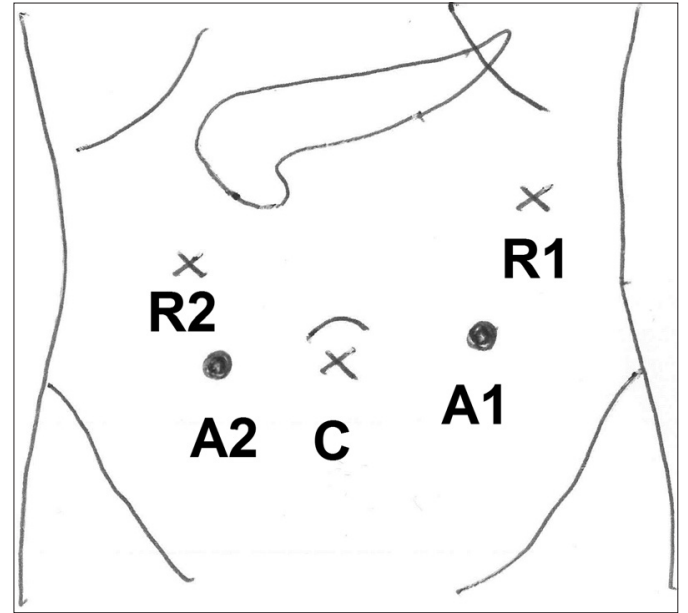

Fig. 1 Diagram shows the positions of the ports for robotic spleen-saving, vessel-preserving distal pancreatectomy. The ports for the camera (C) and robotic arms (R1 and R2), as well as the assistant ports (A1 and A2), are shown.

according to the ISGPF. ${ }^{(25)}$ Complications were graded according to the Clavien-Dindo grading system ${ }^{(26)}$ and recorded up to six months after the operation.

The patient position and port placement were similar for all three patients, with slight variations made according to the position of the tumour. All three patients were placed in the supine, slight reverse Trendelenburg position, with their left side slightly elevated. Open technique (with a 12-mm subumbilical incision) was used to insert the robotic laparoscope. Subsequently, two 8-mm incisions (one in the right hypochondrium/epigastrium and one in the left hypochondrium) were made for the two working arms of the robot. 1-2 assistant ports were subsequently placed; including a 12-mm port in the left iliac fossa for the introduction of an endoscopic stapler, and a 5-mm port in the right hypochondrium for retraction of the liver or elevation of the stomach. The positions of the ports are shown in Fig. 1.

The robot was docked from the patient's head, slightly over the left shoulder. In all three cases, robotic dissection was performed using the robotic monopolar cautery hook, Harmonic ACE® curved shears (Ethicon Inc, Somerville, NJ, USA), Maryland bipolar forceps, fenestrated bipolar forceps and a large needle driver. Conventional laparoscopic instruments were used by the surgical assistant to retract and apply endoclips, aspirate, and apply the endoscopic stapler. The operative technique was similar to that described for conventional LDP. ${ }^{(14)}$ The gastrocolic ligament was opened to access the lesser sac and the splenic flexure was mobilised. Subsequently, the lesion was localised in the pancreas. The inferior border of the pancreas was mobilised via a medial-to-lateral approach, and the splenic artery and vein were identified and isolated. An adequate tunnel was created between the pancreas and the splenic vein, and the pancreas was slung with a nylon tape. The pancreas was divided using an endostapler and oversewn with sutures. Finally, the body and tail of the pancreas were carefully dissected off the splenic artery and vein. Smaller tributaries and branches were divided using the robotic Harmonic curved shears or coagulated using the Maryland bipolar forceps before being divided. Larger tributaries were clipped or suture-ligated. Torn venous tributaries 
Table I. Summary of the clinicopathological features and perioperative outcomes of the three patients who underwent robotic spleen-saving, vessel-preserving distal pancreatectomy.

\begin{tabular}{|c|c|c|c|c|c|c|c|c|c|}
\hline $\begin{array}{l}\text { Case } \\
\text { no. }\end{array}$ & $\begin{array}{l}\text { Age (yr), } \\
\text { gender }\end{array}$ & $\begin{array}{l}\text { Preoperative } \\
\text { diagnosis }\end{array}$ & $\begin{array}{l}\text { Operative } \\
\text { time, } \\
\text { docking } \\
\text { time (min) }\end{array}$ & $\begin{array}{c}\text { Blood } \\
\text { loss }(m L)\end{array}$ & $\begin{array}{l}\text { Pancreatic } \\
\text { texture, } \\
\text { duct size }\end{array}$ & $\begin{array}{c}\text { Postoperative } \\
\text { stay (day) }\end{array}$ & Complication & $\begin{array}{l}\text { Clavien-Dindo } \\
\text { grade }\end{array}$ & Final histology \\
\hline 1 & 64 , female & $\begin{array}{l}2.1-\mathrm{cm} \\
\text { indeterminate } \\
\text { cystic } \\
\text { neoplasm }\end{array}$ & 540,70 & 300 & $\begin{array}{l}\text { Soft, } \\
\text { normal }\end{array}$ & 6 & $\begin{array}{l}\text { Grade A pancreatic } \\
\text { fistula }\end{array}$ & 1 & $\begin{array}{l}\text { Serous cystic } \\
\text { neoplasm }\end{array}$ \\
\hline 2 & 22 , male & $\begin{array}{l}4.5-\mathrm{cm} \text { solid } \\
\text { pseudopapillary } \\
\text { neoplasm }\end{array}$ & 350,15 & 200 & $\begin{array}{l}\text { Soft, } \\
\text { normal }\end{array}$ & 14 & $\begin{array}{l}\text { Grade } 1 \\
\text { postoperative ileus }\end{array}$ & I & $\begin{array}{l}\text { Solid } \\
\text { pseudopapillary } \\
\text { neoplasm }\end{array}$ \\
\hline 3 & 61, female & $\begin{array}{l}1.2-\mathrm{cm} \\
\text { pancreatic } \\
\text { neuroendocrine } \\
\text { tumour }\end{array}$ & 300,25 & 50 & $\begin{array}{l}\text { Soft, } \\
\text { normal }\end{array}$ & 7 & $\begin{array}{l}\text { Grade B pancreatic } \\
\text { fistula requiring } \\
\text { percutaneous } \\
\text { drainage }\end{array}$ & IIIa & $\begin{array}{l}\text { Pancreatic } \\
\text { neuroendocrine } \\
\text { tumour }\end{array}$ \\
\hline
\end{tabular}

were oversewn with Prolene $®$ 4-0 or 5-0 (Ethicon Inc, Cornelia, GA, USA). After resection was completed, biological glue was applied to the pancreatic stump and a closed suction drain was placed in the resection bed. The specimen was removed via Endobag, through the extended subumbilical incision or the assistant port incision.

\section{RESULTS}

The details of the three patients are summarised in Table I. All three cases of robotic SSVP-DP were completed without the need for conversion to the hand-assisted or open approach. The spleen and its main vessels were also successfully conserved in all three cases.

\section{Case 1}

The first case was performed in July 2013 and involved a 64-yearold woman who presented with epigastric pain and dyspepsia. Gastroscopy was performed, but the results were unremarkable. The patient underwent computed tomography (CT) and magnetic resonance (MR) imaging, both of which demonstrated a $2.1-\mathrm{cm}$ solid cystic lesion in the tail of her pancreas (Fig. 2). Endoscopic ultrasonography with fine needle aspirate was performed, but the results were indeterminate. The patient was counselled for surgery due to the indeterminate nature of the cyst. She successfully underwent robotic SSVP-DP. Intraoperatively, the cystic lesion in the tail of the pancreas was found to be partially enveloping the splenic vein. The lesion was successfully dissected off the splenic vein, but with difficulty. Several small tears in the splenic vein were repaired using Prolene 4-0 and 5-0 sutures. The patient's postoperative recovery was uneventful and she was discharged well on postoperative Day 6. She had a biochemical Grade A pancreatic fistula (drain fluid amylase on postoperative Day 3 was $692 \mathrm{IU} / \mathrm{L}$ ), but was otherwise asymptomatic. Final histology demonstrated a serous microcystic neoplasm. The patient was well and pain-free one year after surgery.

\section{Case 2}

The second case involved a 22-year-old man who was incidentally found to have a tumour in the body/neck of the pancreas on CT and intravenous pyelography (Fig. 3), which was performed to test

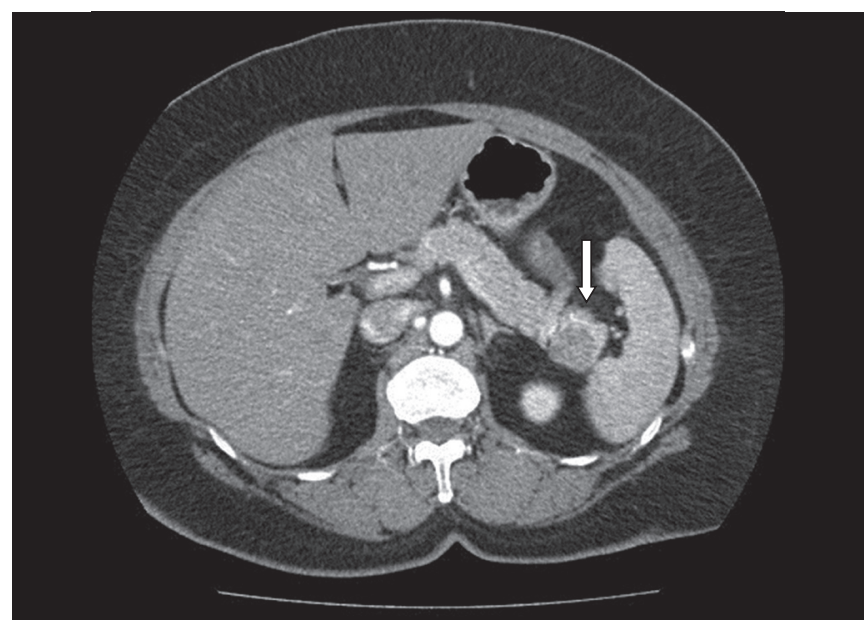

Fig. 2 Case 1: CT image shows an indeterminate cystic lesion (arrow) in the tail of the pancreas.

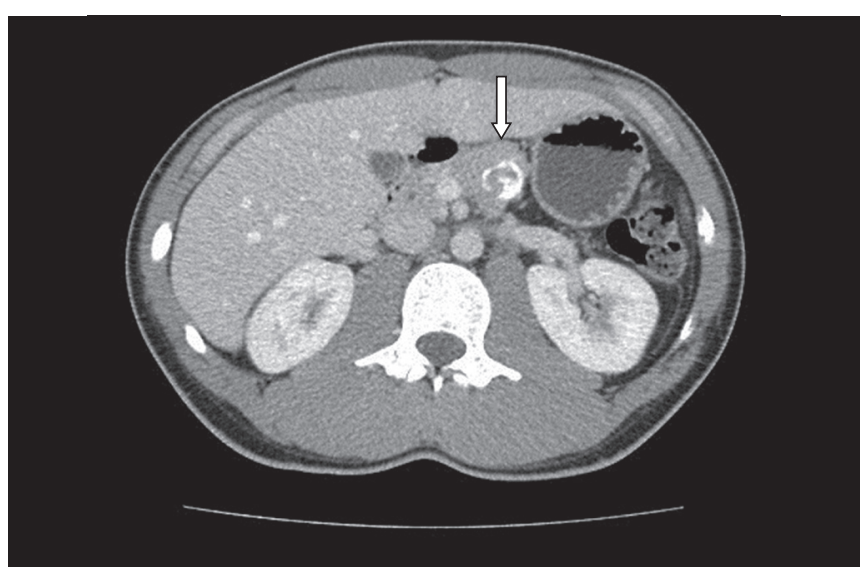

Fig. 3 Case 2: CT image shows a calcified lesion (arrow) in the body/neck of the pancreas.

for haematuria. MR imaging confirmed the presence of a 4.5-cm, partially calcified solid mass that was suspected to be a solid pseudopapillary neoplasm. The patient underwent robotic SSVP-DP in July 2013. His postoperative recovery was complicated by the ileus; the patient experienced abdominal distension, pain and was unable to tolerate a general diet. $\mathrm{CT}$, performed after the operation, 
demonstrated a distended bowel with no transition point. This was managed conservatively and the patient gradually progressed to a soft diet on postoperative Day 7. He was discharged well on postoperative Day 14 with no further complications. Final histology confirmed that the mass was a solid pseudopapillary neoplasm.

\section{Case 3}

The third case involved a 61-year-old woman with diabetes mellitus, hypertension and hyperlipidaemia. A 1.2-cm enhancing neoplasm in the pancreatic tail was incidentally found on CT (Fig. 4), which was performed as part of the investigation for her left lower quadrant abdominal pain. The neoplasm was suspected to be a pancreatic neuroendocrine neoplasm. The patient underwent SSVP-DP in April 2014. Postoperatively, she developed complications from Grade B pancreatic fistula (drain fluid amylase on postoperative Day 3 was 5,067 IU/L). She was initially well and was discharged on postoperative Day 7 , with about 30-60 mL drain fluid a day. She was readmitted on postoperative Day 26 with abdominal discomfort and cloudy drain output. Abdominal CT demonstrated an 8-cm collection and percutaneous drainage was performed. The fluid cultures grew Klebsiella sp. and the patient was treated with appropriate intravenous antibiotics. Her drains were removed on postoperative Day 43 and she was discharged well. She had no further complications and final histology confirmed a Grade 1 pancreatic neuroendocrine neoplasm.

\section{DISCUSSION}

DP is the operation of choice for benign and malignant tumours in the body and tail of the pancreas. Splenectomy is traditionally performed with DP due to the close relationship between the main splenic vessels and the pancreas. ${ }^{(19)}$ However, there is an increasing body of evidence indicating that the spleen plays an important immunological function even in adults; thus, splenic preservation is preferable during the removal of noninvasive, malignant, left-sided pancreatic tumours. ${ }^{(14,19,20)}$ The recent increase in the use of cross-sectional imaging ${ }^{(27)}$ has led to the increased detection of small incidental cystic and solid pancreatic tumours, such as intraductal papillary mucinous neoplasms, ${ }^{(28,29}$ pancreatic neuroendocrine tumours ${ }^{(30)}$ and solid pseudopapillary tumours. ${ }^{(31)}$ These neoplasms are amenable to spleen-saving DP (SS-DP), as they are usually premalignant and not frankly invasive. Nonetheless, the use of SS-DP remains controversial, as it is technically more difficult and may result in longer operation durations and increased blood loss. ${ }^{(32)}$

In order to overcome the difficulty of SS-DP, Warshaw advocated a new technique for SS-DP in 1988, in which the main splenic vein and artery are sacrificed and the spleen is left to perfuse via the short gastric vessels. ${ }^{(21)}$ Although Warshaw's institution reported excellent outcomes with this technique, ${ }^{(33)}$ the results could not be replicated by other institutions, and the technique was reportedly associated with a higher incidence of splenic infarction and left-sided portal hypertension with gastric varices. ${ }^{(19)}$ Hence, many surgeons still prefer to attempt to conserve the main splenic vessels when performing SS-DP;

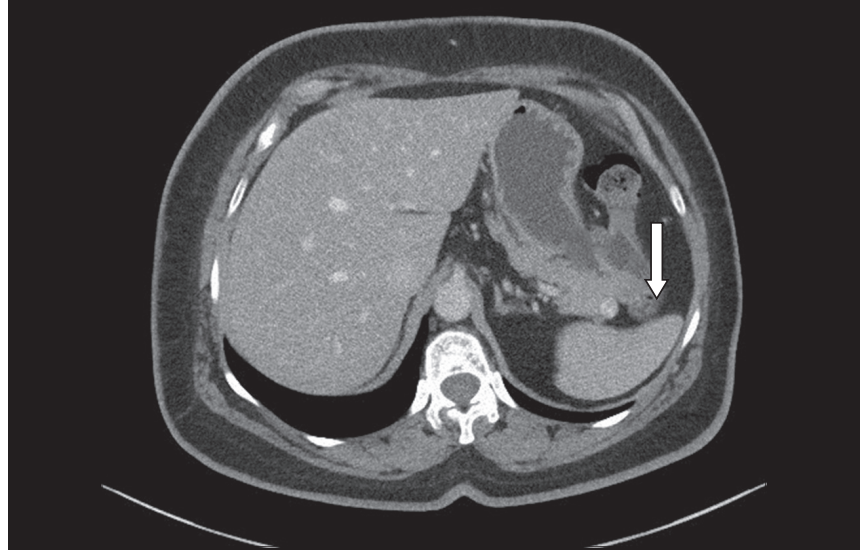

Fig. 4 Case 3: CT image shows a 1.2-cm enhancing solid nodule (arrow) in the tail of the pancreas.

the Warshaw technique is reserved as an intraoperative salvage procedure when it is technically too difficult to separate the splenic vessels from the pancreas (e.g. in patients with pancreatitis). ${ }^{(19,34)}$

Laparoscopic pancreatic surgery was first performed by Cuschieri in 1994. ${ }^{(9)}$ Thereafter, Gagner et al published the first series on LDP in $1996^{(35)}$ and it has been increasingly performed worldwide. Comparisons with open surgery demonstrated that LDP has several advantages, including decreased postoperative pain, faster recovery and decreased blood loss. ${ }^{(11,14,17,36)}$ However, this minimally invasive procedure remains technically demanding, especially when splenic preservation is required; open conversion rates are significant (up to $30 \%$ ) even in highvolume, experienced centres. ${ }^{(16)}$ The rate of splenic preservation has a reported range of $32 \%-84 \%$. $^{(14)}$

To date, the reported use of RDP worldwide is rare. ${ }^{(34)}$ The first series on RDP was reported by Giulianotti et al in 2003. ${ }^{(18)}$ Although there has since been a growing number of RDP cases, the number of case series involving more than 15 patients that report on the outcome of RDP is limited (Table II). ${ }^{(11,17,37-42)}$ The published case series are also limited to centres located in Italy, the United States, China and Korea. The first spleen-saving RDP in Korea was only reported recently in 2011. ${ }^{(34)}$ The rarity of RDP is likely due to limited access to the robotic system and the high cost associated with the procedure. ${ }^{(24,41)}$ Furthermore, despite its theoretical advantages, there is no strong evidence demonstrating the superiority of RDP over the conventional laparoscopic approach. ${ }^{(11)}$ A recent study from Italy, which compared LDP and RDP, reported that RDP was associated with longer operation time but equivalent outcomes. ${ }^{(41)}$ On the other hand, a study from China reported that RDP was associated with shorter operation time and hospital stays. ${ }^{(42)}$ Hence, based on the current evidence, we can only conclude that RDP is feasible and can be performed safely. ${ }^{(7,11,17)}$

However, there is also growing evidence suggesting that RDP may be associated with a shorter learning curve, ${ }^{(15)}$ a lower conversion rate $^{(15)}$ and an increased rate of splenic preservation ${ }^{(11,23,34,38,42)}$ as compared to conventional laparoscopy. In a recent study by Kang et al from Yonsei University, Korea, the authors reported that the rate of splenic preservation was 


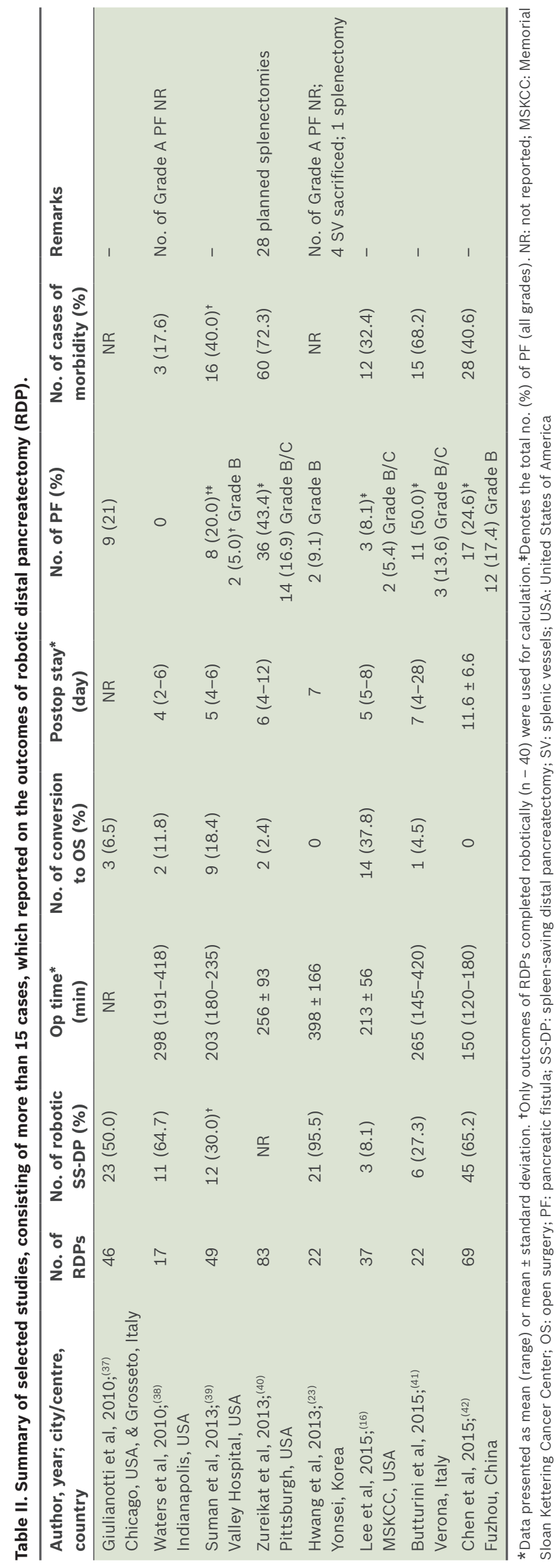

$7 / 16(44 \%)$ for conventional LDP, while that for RDP was $19 / 20$ (95\%; $p=0.027)$. ${ }^{(24)}$ More recently, Daouadi et al (who have the most experience with robotic pancreatectomies worldwide) reported that RDP is superior to conventional LDP, as RDP was associated with a significantly lower conversion rate (RDP: 0/30 vs. LDP: 15/94; p < 0.05) although both methods were equivalent in nearly all measures of outcome and safety. ${ }^{(15)}$ However, it is important to note that the findings of the study by Daouadi et al may have been confounded by various biases. ${ }^{(43)}$ Limitations that are reportedly associated with RDP include longer operation time and greater costs. ${ }^{(11,17,41)}$ Both the study by Kang et al, conducted in Korea, and the study by Butturini et al, conducted in Italy, found that the cost of RDP was more than twice that of LDP and noted that this was the main obstacle to its increased use. ${ }^{(24,41)}$

Currently, DP remains associated with a high morbidity rate, regardless of the surgical approach used. This is despite the fact that most DPs are associated with minor complications and low mortality rates. ${ }^{(12,25,41)}$ The most common complication after DP is the occurrence of a postoperative pancreatic fistulas. ${ }^{(25)}$ The reported rate of this complication is similar for open DP, LDP and RDP; it ranges from $10 \%$ to more than $50 \%$ depending on the definition used. ${ }^{(11,12,17,25,41,44)}$ Thus far, there is no strong evidence that the technique used for parenchymal closure or the operative approach used for DP affects the incidence of pancreatic fistulas. The reported fistula rates are highest in prospective studies that adopt the ISGPF definition, which requires drains to be routinely placed and drain fluid amylase to be routinely measured in all patients, which was the case in the present study. ${ }^{(25,41)}$ Many of the fistulas reported in those studies were Grade A biochemical fistulas, which are completely asymptomatic and do not result in any deviation in the postoperative course of the patient. It is arguable whether these fistulas should even be considered a postoperative complication. Presently, it is uniformly accepted that the risk of fistula formation is increased when the pancreas has a soft texture and the pancreatic duct is small. In the present study, all three patients had high-risk glands and pancreatic fistulas were present in two of the patients; one Grade A and one Grade B fistula, respectively (according to the ISGPF definition). Some investigators have suggested that transaction of the pancreas with specific suture ligation of the main pancreatic duct may decrease the incidence of pancreatic fistulas; however, the supporting evidence remains limited. ${ }^{(12)}$ This practice is also more commonly adopted when DP is performed via the open approach. Currently, stapled transaction of the pancreas is the most common method used to transect the pancreas during LDP. Theoretically, identification and specific ligation of the main pancreatic duct would technically be easier with RDP than with LDP, if the pancreas is transected via an energy device.

Our initial experience with robotic SSVP-DP seems to support the findings in the literature. In all three cases, the spleen and its main vessels were successfully conserved. Although the operative time of robotic SSVP-DP was relatively long, it was comparable to that reported in the literature and could be partly attributed 
to the initial learning curve. A recent study reported that RDP is associated with a learning curve of about 40 cases. ${ }^{(45)}$ However, it is important to emphasise that despite the long operative time, there was minimal blood loss in all three of our patients. We believe that the superiority of the robot in performing fine suturing with Prolene 4-0 and 5-0 sutures in tight spaces likely contributed to our success in conserving the main splenic vessels in all three cases. The high-definition, three-dimensional visualisation, and the increased stability and dexterity of the robotic arms also allowed for more precise dissection of the pancreas from the splenic vasculature. Some of the technical limitations we encountered with the robot includes the lack of tactile feedback (which could be partly overcome by the improved three-dimensional visualisation) and need for an expert surgeon at the bedside for surgical assistance, especially for suction.

In conclusion, our preliminary experience demonstrates that robotic SSVP-DP is feasible in selected patients. Further studies with larger patient cohorts are needed to determine if robotic SSVP-DP is superior to conventional LDP, especially in terms of splenic preservation, rate of conversion to open surgery and cost-effectiveness.

\section{REFERENCES}

1. Purkayastha S, Tilney HS, Georgiou P, et al. Laparoscopic cholecystectomy versus mini-laparotomy cholecysectomy: a meta-analysis of randomised control trials. Surg Endosc 2007; 21:1294-300.

2. Goh BK, Chui CH, Yap TL, et al. Is early laparoscopic appendectomy feasible in children with acute appendicitis presenting with an appendiceal mass? A prospective study. J Pediatr Surg 2005; 40:1134-7.

3. Goh BK, Tan YH, Yip SK, Eng PH, Cheng CW. Outcome of patients undergoing laparoscopic adrenalectomy for primary hyperaldosteronism. JSLS 2004; 8:320-5.

4. Law WL, Lee YM, Choi HK, Seto CL, Ho JW. Impact of laparoscopic resection for colorectal cancer on operative outcomes and survival. Ann Surg 2007; 245:1-7.

5. Goh BK, Chow PK, Chok AY, et al. Impact of the introduction of laparoscopic wedge resection as a surgical option for suspected small/medium-sized gastrointestinal stromal tumors of the stomach on perioperative and oncologic outcomes. World J Surg 2010; 34:1847-52.

6. Koh $\mathrm{YX}$, Chok AY, Zheng HL, et al. A systematic review and meta-analysis comparing laparoscopic versus open gastric resections for gastrointestinal stromal tumors of the stomach. Ann Surg Oncol 2013; 20:3549-60.

7. Hanna EM, Rozario N, Rupp C, et al. Robotic hepatobiliary and pancreatic surgery: lessons learned and predictors for conversion. Int J Med Robot 2013; 9:152-9.

8. Goh BK, Chan CY, Wong JS, et al. Factors associated with and outcomes of open conversion after laparoscopic minor hepatectomy: initial experience at a single institution. Surg Endosc 2015; 29:2636-42.

9. Cuschieri A. Laparoscopic surgery of the pancreas. J R Coll Surg Edinb 1994; 39:178-84.

10. Cherqui D, Husson E, Hammoud R, et al. Laparoscopic liver resections: a feasibility study in 30 patients. Ann Surg 2000; 232:753-62.

11. Strijker M, van Santvoort HC, Besselink MG, et al. Robot-assisted pancreatic surgery: a systematic review of the literature. HPB (Oxford) 2013; 15:1-10.

12. Goh BK, Tan YM, Chung YF, et al. Critical appraisal of 232 consecutive distal pancreatectomies with emphasis on risk factors, outcome and management of the post-operative pancreatic fistula: a 21-year experience at a single institution. Arch Surg 2008; 143:956-65.

13. Cameron JL, Riall TS, Coleman J, Belcher KA. One thousand consecutive pancreaticoduodenectomies. Ann Surg 2006; 244:10-5.

14. lacobone M, Citton M, Nitti D. Laparoscopic distal pancreatectomy: up-to-date and literature review. World J Gastroenterol 2012; 18:5329-37.

15. Daouadi M, Zureikat A, Zenati MS, et al. Robot-assisted minimally invasive distal pancreatectomy is superior to the laparoscopic technique. Ann Surg 2013; 257:128-32.

16. Lee SY, Allen PJ, Sadot E, et al. Distal pancreatectomy: a single institution's experience in open, laparoscopic, and robotic approaches. J Am Coll Surg 2015; 220:18-27.
17. Cirocchi R, Partelli S, Coratti A, et al. Current status of robotic distal pancreatectomy: a systematic review. Surg Oncol 2013; 22:201-7.

18. Giulianotti PC, Coratti A, Angelini M, et al. Robotics in general surgery: personal experience in a large community hospital. Arch Surg 2003; 138:777-84

19. Lee SY, Goh BK, Tan YM, et al. Spleen-preserving distal pancreatectomy. Singapore Med J 2008; 49:883-5.

20. Shoup M, Brennan MF, McWhite K, et al. The value of splenic preservation with distal pancreatectomy. Arch Surg 2002; 137:164-8.

21. Warshaw AL. Conservation of the spleen with distal pancreatectomy. Arch Surg $1988 ; 123: 550-3$

22. Hwang HK, Chung YE, Kim KA, Kang CM, Lee WJ. Revisiting vascular patency after spleen-preserving laparoscopic distal pancreatectomy with conservation of splenic vessels. Surg Endosc 2012; 26:1765-71.

23. Hwang HK, Kang CM, Chung YE, et al. Robot-assisted spleen-preserving distal pancreatectomy: a single surgeon's experiences and proposal of clinical application. Surg Endosc 2013; 27:774-81.

24. Kang CM, Kim DH, Lee WJ, Chi HS. Conventional laparoscopic and robotassisted spleen-preserving pancreatectomy: does da Vinci have clinical advantages. Surg Endosc 2011; 25:2004-9.

25. Bassi C, Dervenis C, Butturini G, et al; International Study Group on Pancreatic Fistula Definition. Postoperative pancreatic fistula: an international study group (ISGPF) definition. Surgery 2005; 138:8-13.

26. Dindo D, Demartines N, Clavien PA. Classification of surgical complications: a new proposal with evaluation in a cohort of 6,336 patients and results of a survey. Ann Surg 2004; 240:205-13.

27. Goh BK, Tan YM, Cheow PC, et al. Cystic lesions of the pancreas: an appraisal of an aggressive resectional policy adopted at a single institution during 15 years. Am J Surg 2006; 192:148-54.

28. Goh BK, Tan YM, Thng $\mathrm{CH}$, et al. How useful are clinical, biochemical, and cross-sectional imaging features in predicting potentially malignant or malignant cystic lesions of the pancreas? Results from a single institution experience with 220 surgically treated patients. J Am Coll Surg 2008; 206:17-27.

29. Goh BK, Thng CH, Tan DM, et al. Evaluation of the Sendai and 2012 International Consensus Guidelines based on cross-sectional imaging findings performed for the initial triage of mucinous cystic lesions of the pancreas: a single institution experience with 114 surgically treated patients. Am J Surg 2014; 208:202-9.

30. Goh BK, Chow PK, Tan YM, et al. Validation of five contemporary prognostication systems for primary pancreatic endocrine neoplasms: results from a single institution experience with 61 surgically-treated cases. ANZ J Surg 2011; 81:79-85.

31. Goh BK, Tan YM, Cheow PC, et al. Solid pseudopapillary neoplasms of the pancreas: an updated experience. J Surg Oncol 2007; 95:640-4.

32. Aldridge MC, Williamson RC. Distal pancreatectomy with and without splenectomy. Br J Surg 1991; 78:976-9.

33. Ferrone CR, Konstantinidis IT, Sahani DV, et al. Twenty-three years of the Warshaw operation for distal pancreatectomy with preservation of the spleen. Ann Surg 2011; 253:1136-9.

34. Kim DH, Kang CM, Lee WJ, Chi HS. The first experience of robot-assisted spleen-preserving laparoscopic distal pancreatectomy in Korea. Yonsei Med J $2011 ; 52: 539-42$

35. Gagner M, Pomp A, Herrera MF. Early experience with laparoscopic resections of islet cell tumors. Surgery 1996; 120:1051-4.

36. Pasupathy S, Goh BK, Shrikhande SV, Mesenas SJ. Is there a place for N.O.T.E.S. in the diagnosis and treatment of neoplastic lesions of the pancreas? Surg Oncol 2009; 18:139-46.

37. Giulianotti PC, Sbrana F, Bianco FM, et al. Robot-assisted laparoscopic pancreatic surgery: single-surgeon experience. Surg Endosc 2010; 24:1646-57.

38. Waters JA, Canal DF, Wiebke EA, et al. Robotic distal pancreatectomy: cost effective? Surgery 2010; 148:814-23.

39. Suman P, Rutledge J, Yiengpruksawan A. Robotic distal pancreatectomy. JSLS $2013 ; 17: 627-35$

40. Zureikat A, Moser AJ, Boone BA, et al. 250 robotic pancreatic resections: safety and feasibility. Ann Surg 2013; 258:554-9.

41. Butturini G, Damoli I, Crepaz L, et al. A prospective non-randomised singlecenter study comparing laparoscopic and robotic distal pancreatectomy. Surg Endosc 2015; 29:3163-70

42. Chen S, Zhan Q, Chen JZ, et al. Robotic approach improves spleen-preserving rate and shortens postoperative hospital stay of laparoscopic distal pancreatectomy: a matched cohort study. Surg Endosc 2015; 29:3507-18.

43. Goh BK. Robot-assisted minimally invasive distal pancreatectomy is superior to the laparoscopic technique. Ann Surg 2016; 263:e49.

44. Goh BK, Tan YM, Cheow PC, et al. Outcome of distal pancreatectomy for pancreatic adenocarcinoma. Dig Surg 2008; 25:32-8.

45. Shakir M, Boone BA, Polanco PM, et al. The learning curve for robotic distal pancreatectomy: an analysis of outcomes of the first 100 consecutive cases at a high-volume pancreatic centre. HPB (Oxford) 2015; 17:580-6. 